\title{
Effectiveness and Therapeutic Impact of CT-Guided Percutaneous Drainage for Deep Neck Abscesses
}

\author{
Zexing Cheng1, Xiaoming Tang², Juebo Yu ${ }^{*}$ \\ ${ }^{1}$ Department of Otolaryngology, Yangzhou First People's Hospital, Yangzhou, China \\ ${ }^{2}$ Department of Computed Tomography and Interventional Radiology, Yangzhou First People's Hospital, \\ Yangzhou, China \\ Email: "yujuebo2004@163.com
}

Received 20 October 2015; accepted 16 November 2015; published 19 November 2015

Copyright (C) 2015 by authors and Scientific Research Publishing Inc.

This work is licensed under the Creative Commons Attribution International License (CC BY). http://creativecommons.org/licenses/by/4.0/

(c) (i) Open Access

\begin{abstract}
Objective: The purpose of this study is to evaluate the effectiveness and safety of CT-guided percutaneous drainage (CPD) in the management of deep neck abscesses. Factors associated with successful treatment in patients with DNA will be identified. Methods: We retrospectively studied 29 patients who presented to the department of otolaryngology with deep neck abscesses between April 2011 and April 2015. These 29 patients were managed with CPD after antibiotic therapy or needle aspiration failed. Data on patient demographics, location of infection, existing comorbidity, duration of hospitalization, treatment received, and complications were reviewed. Results: The average age of 29 patients, including 18 men and 11 women, was 56 years old. Abscess was found in parapharyngeal space $(n=16)$, submandibular space $(n=7)$, retropharyngeal space $(n=5)$ and pretracheal space $(n=1)$. The maximum transverse diameter of abscess ranged from $4.8 \mathrm{~cm}$ to 8.0 cm (mean $6.03 \mathrm{~cm}$ ). Positive cultures were found in 24 cases and the most common pathogen found was Streptococcus viridans. Average hospital stay was 6.7 days. Deep neck abscesses were completely removed without residual in all patients. No one had complications and no one died during and after CPD. Conclusion: CPD is a safe and highly effective procedure for treating patients with deep neck abscesses who do not respond to antibiotics therapy. This technique can also provide reliable evidence on pathogens responsible for deep neck abscesses and help otolaryngologists choose effective treatment to achieve better clinical success rate. We recommend that most deep neck abscesses should be managed initially by CPD before resorting to open surgery.
\end{abstract}

\section{Keywords}

Deep Neck Abscesses, CT-Guided Percutaneous Drainage, Abscess

\footnotetext{
${ }^{*}$ Corresponding author.
}

How to cite this paper: Cheng, Z.X., Tang, X.M. and Yu, J.B. (2015) Effectiveness and Therapeutic Impact of CT-Guided Percutaneous Drainage for Deep Neck Abscesses. International Journal of Otolaryngology and Head \& Neck Surgery, 4, 409416. http://dx.doi.org/10.4236/ijohns.2015.46066 


\section{Introduction}

Deep neck abscesses are defined as collections of pus contained within fascial planes and spaces of head and neck. Abscesses are likely to expand from one neck space to another. Clinical symptoms will vary, depending on the exact region and dimensions of abscess spread [1]. Simple upper respiratory infection or dental infection may ultimately progress to deep neck abscesses, with potentially life-threatening complications such as upper airway obstruction, mediastinitis, internal jugular vein thrombosis, septic shock, and death [2] [3]. Although the prevalence and complication incidence of deep neck abscesses have been diminished due to improved diagnostic techniques and widespread use of antibiotics, deep neck abscesses continue to cause significant morbidity and mortality [4]-[6].

Open surgical incision-and-drainage procedure with appropriate administration of antibiotics remains the mainstay of treatment for deep neck abscesses. However, it usually involves neck incisions and exploration, which exposes patients to a cosmetically undesirable scar and a risk of neurovascular injury. Moreover, open surgery is the most expensive treatment that usually requires general anesthesia and hospitalization. CT-guided percutaneous drainage (CPD) has been used recently to remove abscesses in patients who do not have airway compromise [1] [7]. CPD has several advantages including small point of entry, quick healing time, little or no scar formation, and lower risk of contaminating surrounding deep neck spaces. Some studies have suggested that CPD of deep neck abscesses is a less invasive and an effective alternative to open surgery in select cases [1] [7].

This study presents a four-year experience of using CPD to treat deep neck abscesses at our institution. The primary objective of this study is to evaluate the effectiveness and safety of CPD for deep neck abscesses and to determine the factors influencing clinical success.

\section{Methods}

\subsection{Data Collection}

Medical and radiological record of all patients with deep neck abscesses who underwent CPD between April 2011 and April 2015 at the department of otolaryngology of one large tertiary hospital in Yangzhou, China, were reviewed. This study was approved by Research Ethics Committee of the hospital. Patients were excluded if: they did not provide informed consent; they were pregnant; they had peritonsillar abscesses; they had superficial abscesses; they had head or neck tumors; they had multi-lobulated or ill-defined abscess; or they had recurrent neck abscesses. Altogether, 29 patients were included in this study. Information reviewed included: patient age and gender, location of infection, existing comorbidity, duration of hospitalization, treatment received and complications.

\subsection{CT-Guided Percutaneous Drainage (CPD) Procedure}

The decision for CPD was made if patients were in one of the following conditions: there were impending complications; no improvement was observed 48 hours after antibiotics were administered; collection of pus was found within fascial spaces or a glandular structure; lobulated abscesses were greater than $3 \mathrm{~cm}$ in diameter or extended into deep neck spaces.

All drainage procedures were performed with CT guidance by an interventional radiologist and an attending doctor of the ward or a resident of the department of radiology. In order to choose the safest pathway for insertion of catheter and to avoid possible injury to neighboring vital structures, radiologists with more than 10 years' experience were invited to perform the procedure.

All procedures used pigtail catheter tubes. The size of catheter, either $10 \mathrm{~F}$ or $12 \mathrm{~F}$, depended on the maximum transverse diameter of patients' abscesses. The exact skin entry site was defined from the contrast-enhanced CT (CECT). A trocar-type pigtail catheter, consisting of a pigtail drainage catheter and an appropriately sized twopart trocar needle, was inserted into the abscess cavity after the puncture site was sterilely prepared and anesthetized. The catheter was secured in place by suturing. Immediately after insertion of the pigtail catheter, a $20 \mathrm{ml}$ syringe was mounted on the catheter and the abscess contents were aspirated from the abscess cavity. The pus was sent for gram stain and cultures in order to identify the pathogenetic organism responsible for abscess. Then, the pigtail catheter was connected to a vacuum suction ball for continuous drainage. Flushing the tube with 15 $\mathrm{ml}$ of sterile saline and metronidazole once daily was usually prescribed to assure patency of the tube. It should be noted that complete evacuation by aspiration of the abscess cavity was not performed on CT table because it may result in severe hemoptysis from carotid artery rupture. 


\subsection{Follow-Up after CPD Procedure}

CT images were taken repeatedly to confirm the correct catheter placement. Patients were firstly administered empiric parenteral antibiotics and then specific antibiotics with or without intravenous steroids based on their drug sensitivity results. Day to day follow-up of patients was done by the referring physician, including clinical observations, daily catheter output measurements, and repeated ultrasound examination and repeated CECT when necessary. Catheter was left in place until drainage of pus from the catheter stopped or decision of surgery was taken due to non-closure of the abscess cavity. After discharge from hospital, patients were asked to take oral antibiotics tailored to their antibiograms for at least 14 days. All patients were followed up regularly by clinical presentation or imaging for at least 6 months.

Technical success was defined as appropriate placement of pigtail catheter into the target abscess cavity, with confirmation by means of subsequent aspiration of pus and CT scan control. Clinical treatment success was recorded when the following criteria were met: control of sepsis, resolution of abscess cavity on imaging, and no requirement for open surgical incision-and-drainage.

\section{Results}

Demographics, medical history and clinical outcomes of all patients reviewed are summarized in Table 1. Among 29 patients with uniloculated neck abscesses, 16 of them had abscesses located in the parapharyngeal space (Figure 1), 7 in retropharyngeal space (Figure 2), 5 in submandibular space (Figure 3 ) and 1 in the pretracheal space. CECT findings revealed airway compression in 8 patients. The maximum transverse diameter of abscess ranged from $4.8 \mathrm{~cm}$ to $8.0 \mathrm{~cm}$.

Of 29 patients undergoing CPD, immediate technical success was achieved in all patients with precise placement of catheter in deep neck abscess cavities. Direct trocar technique was applied in all cases. 10F pigtail catheter was used in 16 patients whose abscess diameter was between $3 \mathrm{~cm}$ and $6 \mathrm{~cm}$. $12 \mathrm{~F}$ pigtail catheter was used

Table 1. Patient demographics, medial history and clinical outcomes.

\begin{tabular}{|c|c|}
\hline Variables & CT-guided percutaneous drainage $(n=29)$ \\
\hline \multicolumn{2}{|l|}{ Demographics } \\
\hline Mean age & $56(13.4)$ \\
\hline Male sex (\%) & $18(62.1)$ \\
\hline \multicolumn{2}{|l|}{ Underlying diseases } \\
\hline Diabetes mellitus (\%) & $7(24.1)$ \\
\hline \multicolumn{2}{|l|}{ Clinical outcomes } \\
\hline Mean maximum transverse diameter of abscess $(\mathrm{cm})$ & $6.03(0.79)$ \\
\hline Mean catheter period (days) & $4.8(1.22)$ \\
\hline Mean hospital stay (days) & $6.7(1.52)$ \\
\hline \multicolumn{2}{|l|}{ Pathogen isolated } \\
\hline \multicolumn{2}{|l|}{ Aerobic } \\
\hline Streptococcus viridans & 9 \\
\hline Staphylococcus aureus & 5 \\
\hline Klebsiella pneumoniae & 4 \\
\hline Escherichia coli & 3 \\
\hline Haemophilus influenzae & 2 \\
\hline \multicolumn{2}{|l|}{ Anaerobic } \\
\hline Peptostreptococcus sp. & 3 \\
\hline Bacteroides sp. & 1 \\
\hline
\end{tabular}

Data are number (SD) except where otherwise specified. SD = standard deviation. 


\section{Z. X. Cheng et al.}

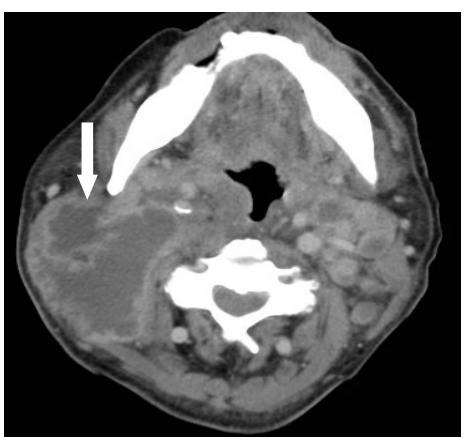

(a)

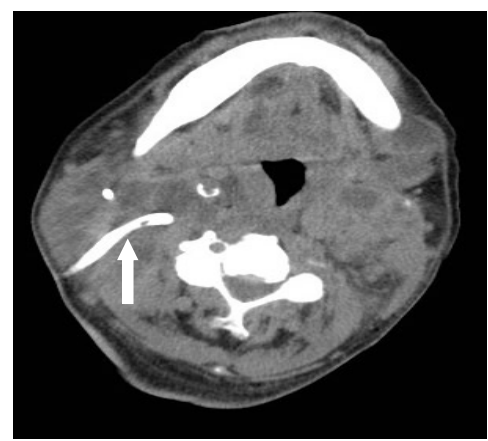

(b)

Figure 1. Images from a 48-year-old man with right a paralaryngeal abscess measuring $6.5 \mathrm{~cm} \times 7.5 \mathrm{~cm} \times 8.0 \mathrm{~cm}$, successfully treated with catheter drainage. (a) Right parapharyngeal space low attenuation abscess with contrast enhancing rim and internal septation lying deep to edematous sternocleidomastoid muscle with vascular structures lying anteromedial (arrowhead); (b) Cervical CT without contrast enhancement demonstrated the right paralaryngeal abscess with the pigtail catheter in situ (arrowhead).

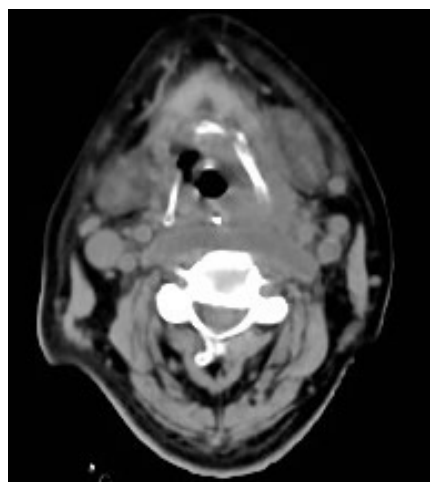

(a)

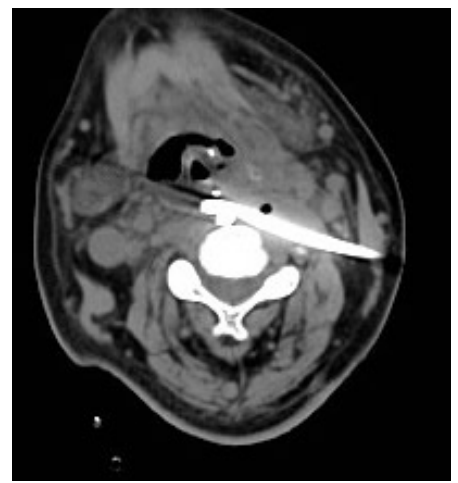

(b)

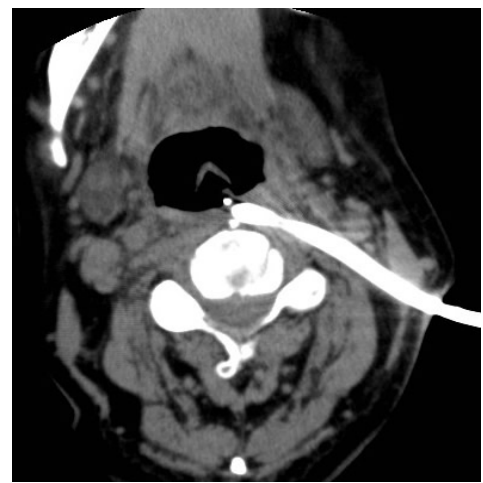

(c)

Figure 2. Images from a 67-year-old man with a retropharyngeal abscess measuring $2.2 \mathrm{~cm} \times 6.2 \mathrm{~cm} \times 8.0 \mathrm{~cm}$, successfully treated with catheter drainage. (a) Contrast-enhanced neck CT reveals a uniloculated abscess (arrowhead) extending from the retropharyngeal space anterior laterally into the parapharyngeal space; (b) The trocar pigtail catheter (arrowhead) has been inserted into the abscess cavity; (c) Cervical CT without contrast enhancement taken 4 days after initial catheter drainage. An $12-\mathrm{F}$ pigtail catheter (arrows) is seen within a retropharyngeal abscess cavity successfully draining the deep retropharyngeal component. No obvious pus is seen.
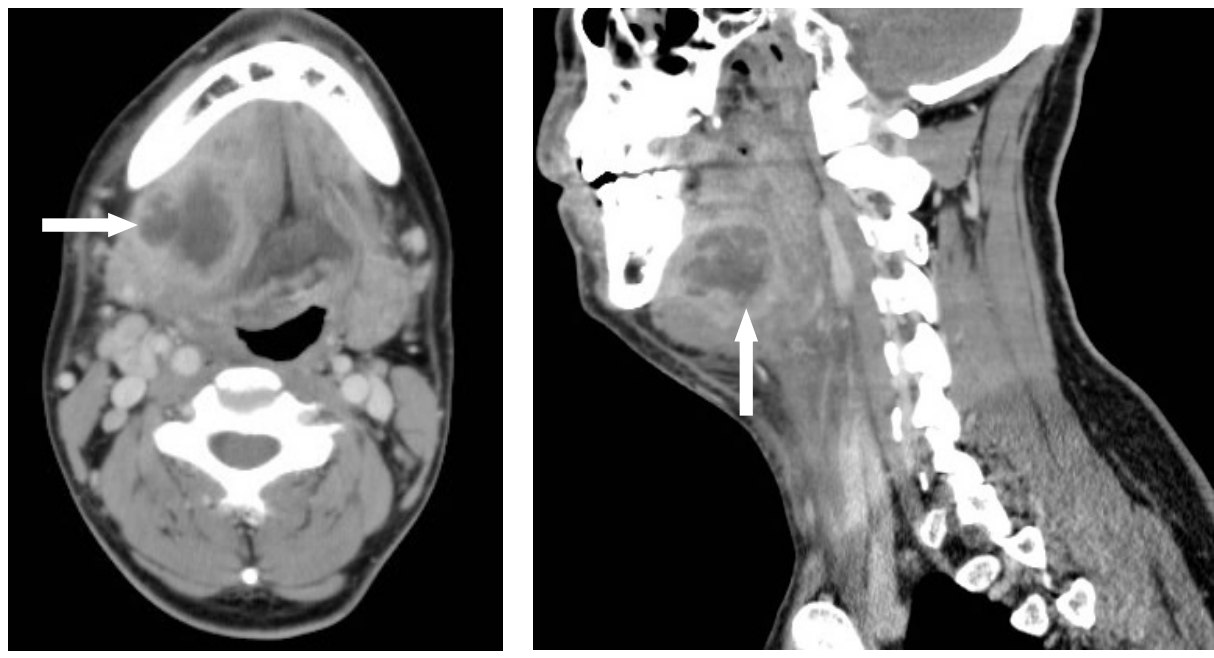

(a) 


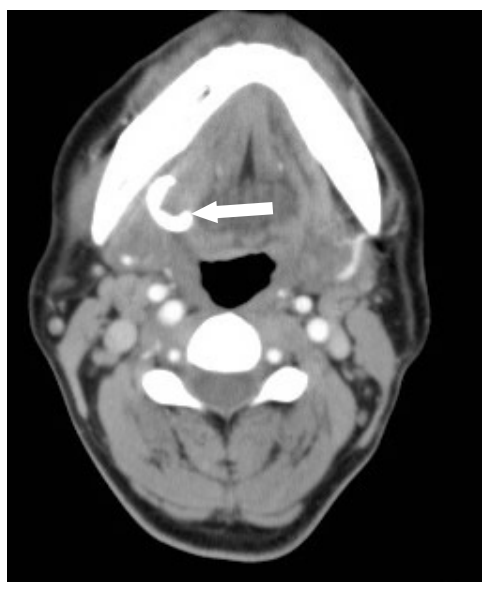

(b)

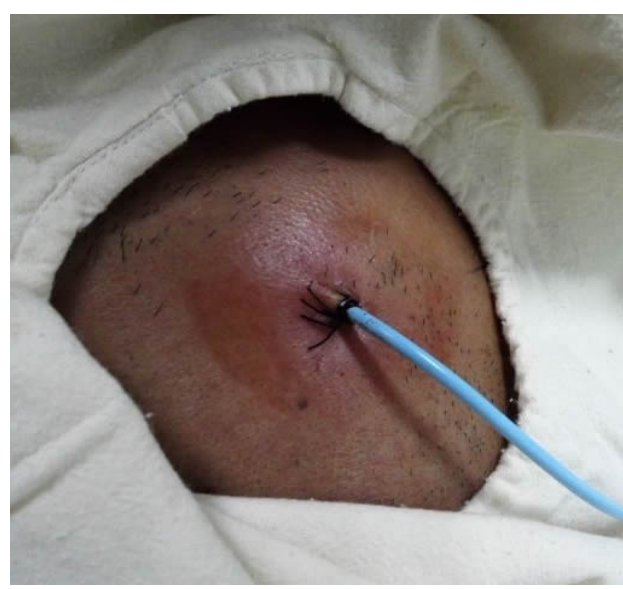

(c)

Figure 3. (a) Cervical CT (axial and sagittal reconstruction) showing a right submandibular abscess measuring $5.5 \mathrm{~cm} \times 5.5$ $\mathrm{cm} \times 5.0 \mathrm{~cm}$, located on the right side and reaching the midline, which also compressed and displaced the airway at the level of the oropharynx; (b) Percutaneous catheter in place there day after evacuation of the right parapharyngeal abscess and antibiotic treatment. No obvious pus is seen; (c) The patient after positioning of the catheter.

in 13 patients whose abscess diameter was greater than $6 \mathrm{~cm}$. The procedure was well tolerated by all patients. The mean procedure time was 20 minutes. None of those patients required open incision-and-drainage surgery afterwards. Clinical success was achieved in all patients as well. After CPD, no patient required surgical treatment for uncontrolled infections, or died of infections.

At follow-up CT scan, all 29 patients who underwent CPD showed complete drainage of abscesses. The indwelling catheter period ranged from 3 to 7 days. Patients spent 6.7 days in hospital on average. Patients with diabetes mellitus (DM) had longer hospital stay than those without DM (8.6 days vs. 6.1 days) and the difference between two groups was significant $(\mathrm{p}<0.05)$. Catheters were removed when the abscess cavity had been completely drained and symptoms such as a swollen neck, fever and pain had disappeared.

Cultures of aspirated pus were positive in 24 out of 29 patients (82.8\%). Pathogens found in aspirated pus were either aerobic or anaerobic (Table 1). Multiple pathogens were found in four samples of abscesses.

After treatment, all patients were cured of their disease completely and no complications were detected. After discharge from hospital, no patient showed symptoms or signs of relapse at ultrasound follow-up.

\section{Discussion}

The widespread availability of antibiotics has reduced the incidence of deep neck abscesses drastically [2] [3]. However, deep neck abscesses remains a severe condition as it may potentially lead to life-threatening situations, especially when there is a delay in diagnosis or treatment. Surgery such as incision-and-drainage procedure bears the risk of neurovascular injury and leaving a scar. As a result, less invasive procedure has been investigated for its effectiveness and safety. In this study, we performed a retrospective study on patients who underwent CPD for deep neck abscesses. The results showed that CPD was both technically and clinically successful for all patients. No complications or relapse were reported after patients were discharged from hospital.

Clinical diagnosis of deep neck abscesses was mainly based on clinical examination and imaging examination. CT scan, especially CECT, is an important tool for evaluation of the lesion. It can be used in all patients to establish diagnosis and provide valuable information to help disclose the origin, location (also in relation to the major vascular structures and the airway), and extent of deep neck abscesses. CECT is essential in distinguishing an abscess from cellulitis or a phlegmon [8]. CECT provides a wider field of view and is more useful than ultrasound scan in defining the extent of neck abscesses as ultrasound scan cannot always identify small or deep abscess and cannot provide the specific anatomical information necessary for surgical intervention [9].

Therapeutic management of deep neck abscesses includes rational antibiotic treatment, drainage (surgical or percutaneous), repeated needle aspiration, and open surgery [1]. Recent studies have shown that, in select cases, uncomplicated deep neck abscesses can be effectively treated with antibiotics and careful monitoring, without surgical drainage [10] [11]. However, Surgical or percutaneous drainage is required in $10 \%$ to $83 \%$ of patients 
who presented with deep neck space infections [12] [13], as medical therapy could fail due to virulence of the responsible pathogens or inadequate concentration of antibiotics within the abscess cavity [10] [14]. The open surgical drainages are effective but have some significant disadvantages. The patients are required to have an operating room and team and a general anesthetic, which may also necessitate securing of airway fiber optically or with a tracheotomy. The open neck wounds may also add the risk of neurovascular injury and result in a cosmetically undesirable scar.

CPD has been proved to be safe and effective with less morbidity and mortality than surgical resection [15] [16]. It can improve the accuracy in reaching the involved site and decrease costs for a non-operating room procedure. Other advantages of CT-guided percutaneous drainage are rapid clinical and radiological improvement of pyogenic abscess which may avoid complications that can occur with prolonged, improved cosmetic results and conservative treatment. Therefore, it can be used as an alternative to surgical drainage.

Although ultrasound and CT scanning guidance were the most common techniques used for placement of needles or catheters, CT-guided drainage was chosen in this study. There are several reasons. First of all, CT scan is a useful diagnostic tool to detect and establish the treatment plan for deep neck abscesses. CECT is able to identify impeding airway complications before they present clinically [17]. Second, CT can differentiate abscess from carotid artery aneurysm, cellulitis or phlegmon, which is important since catheter drainage has a higher complication rate. Third, CT is optimal in determining the wall thickness of an abscess, contents of an abscess and its relationship to the adjacent neck vital structures. Moreover, any obstructing foreign body or head and neck neoplasms can also be visualized. Finally, when a deeper abscess is encountered, CT-guided needle aspiration is more feasible than ultrasound guidance [16].

Previous studies have demonstrated that CPD showed high success rate for infection treatment and saved patients from more invasive surgical intervention. In our study, none of the patients required any further surgical intervention without any complications related to drainage procedures. In addition, clinical success rate was extremely high $(100 \%, n=29)$, which is similar to prior studies, and no complications occurred. These results are comparable to the results of the largest series published to date. Cole et al. [7] reported successful CPD in 2 patients without complications, and surgery was avoided in these 2 patients (100\% success rate). Thanos et al. [1] also reported successful CT-guided percutaneous catheter drainage of potentially life-threatening neck abscesses in 15 patients and surgery was avoided in 14 patients (93\% success rate). Surgery was performed in one patient because of the presence of multiple internal septation that could not be drained via percutaneous catheters. No complications were reported in their study.

In this study, we used the trocar technique for pigtail catheters insertion in all cases. Although some other literature suggested that the Seldinger technique with placement of catheter might decreases the likelihood of complications [16], we believed that the choice of technique depended on the ability, experience and preference of the interventional radiologist performing the procedure. In our study, we used $10 \mathrm{~F}$ and $12 \mathrm{~F}$ catheters for drainage based on the size of deep neck abscesses and irrigated the abscess cavities with metronidazole and saline solution until the wash out was clear. The duration of purulent discharge varied from 3 to 9 days. We used a vacuum suction ball for continuous drainage to facilitate the evacuation of residual pus which is similar to Chang et al. study [18]. The main advantages of this closed drainage system are the superior and persistent negative pressure for drainage, prevention of contamination in the surgical dressing, and easy management for nursing [19]. We obtained positive cultures in $82.8 \%$ of cases, which is consistent with other studies where positive cultures ranged from $56.3 \%-85.7 \%$ of deep neck abscesses cases [1] [11] [20]. Streptococcus species were found in the majority of cases (48.2\%), which is also consistent with other studies of deep neck abscesses [21] [22]. Our study results suggest that CPD with bacteria cultures can help us make more rational decision on treatment for infections.

\section{Limitation}

The limitation of this study should be acknowledged. First of all, we included a very small sample of patients in our review. Besides, retrospective studies may be less reliable in terms of data collection. Prospective study and more cases are required to further examine the effectiveness of CPD in the future.

\section{Conflict of Interest}

The authors declare no conflict of interest. 


\section{References}

[1] Thanos, L., Mylona, S., Kalioras, V., et al. (2005) Potentially Life-Threatening Neck Abscesses: Therapeutic Management under CT-Guided Drainage. CardioVascular and Interventional Radiology, 29, 196-199.

http://dx.doi.org/10.1007/s00270-004-0003-y

[2] Eftekharian, A., Roozbahany, N.A., Vaezeafshar, R., et al. (2009) Deep Neck Infections: A Retrospective Review of 112 Cases. European Archives of Oto-Rhino-Laryngology, 266, 273-277. http://dx.doi.org/10.1007/s00405-008-0734-5

[3] Hasegawa, J., Hidaka, H., Tateda, M., Kudo, T., Sagai, S., Miyazaki, M., et al. (2010) An Analysis of Clinical Risk Factors of Deep Neck Infection. Auris Nasus Larynx, 10, 356-361.

[4] Ridder, G.J., Technau-Ihling, K., Sander, A., et al. (2005) Spectrum and Management of Deep Neck Space Infections: An 8-Year Experience of 234 Cases. Otolaryngology—Head \& Neck Surgery, 133, 709-714.

http://dx.doi.org/10.1016/j.otohns.2005.07.001

[5] Huang, T.T., Liu, T.C., Chen, P.R., Tseng, F.Y., Yeh, T.H. and Chen, Y.S. (2004) Deep Neck Infection: Analysis of 185 Cases. Head \& Neck, 26, 854-860. http://dx.doi.org/10.1002/hed.20014

[6] Bottin, R., Marioni, G., Rinaldi, R., et al. (2003) Deep Neck Infection: A Present-Day Complication. A Retrospective Review of 83 Cases (1998-2001). European Archives of Oto-Rhino-Laryngology, 260, 576-579. http://dx.doi.org/10.1007/s00405-003-0634-7

[7] Cole, D., Bankoff, M. and Carter, B. (1984) Percutaneous Catheter Drainage of Deep Neck Infections Guided by CT. Radiology, 152, 224. http://dx.doi.org/10.1148/radiology.152.1.6729120

[8] Marioni, G., Castegnaro, E., Staffieri, C., et al. (2006) Deep Neck Infection in Elderly Patients. A Single Institution Experience (2000-2004). Aging Clinical and Experimental Research, 18, 127-132. http://dx.doi.org/10.1007/BF03327427

[9] Smith II, J.L., Hsu, J.M. and Chang, J. (2006) Predicting Deep Neck Space Abscess Using Computed Tomography. American Journal of Otolaryngology, 27, 244-247. http://dx.doi.org/10.1016/j.amjoto.2005.11.008

[10] McClay, J.E., Murray, A.D. and Booth, T. (2003) Intravenous Antibiotic Therapy for Deep Neck Abscesses Defined by Computed Tomography. Archives of Otolaryngology—Head \& Neck Surgery, 129, 1207-1212. http://dx.doi.org/10.1001/archotol.129.11.1207

[11] Lee, Y.Q. and Kanagalingam, J. (2011) Deep Neck Abscesses: The Singapore Experience. European Archives of Oto-Rhino-Laryngology, 268, 609-614. http://dx.doi.org/10.1007/s00405-010-1387-8

[12] Mayor, G.P., Milan, J.M. and Martinez-Vidal, A. (2001) Is Conservative Treatment of Deep Neck Space Infections Appropriate? Head \& Neck, 23, 126-133. http://dx.doi.org/10.1002/1097-0347(200102)23:2<126::AID-HED1007>3.0.CO;2-N

[13] Ridder, J.G., Eglinger, C.F., Technau-Ihlinge, K., et al. (2000) Deep Neck Abscess Masquerading Hypopharyngeal Carcinoma. Otolaryngology—Head and Neck Surgery, 123, 659-660. http://dx.doi.org/10.1067/mhn.2000.110613

[14] Oh, J.-H., Kim, Y. and Kim, C.-H. (2007) Parapharyngeal Abscess: Comprehensive Management Protocol. ORL, 69, 37-42. http://dx.doi.org/10.1159/000096715

[15] Poe, L.B., Petro, G.R. and Matta, I. (1996) Percutaneous CT-Guided Aspiration of Deep Neck Abscesses. AJNRAmerican Journal of Neuroradiology, 17, 1359-1363.

[16] Cottrell, D.A., Bankoff, M. and Norris, L.H. (1992) Computed Tomography-Guided Percutaneous Drainage of a Head and Neck Infection. Journal of Oral and Maxillofacial Surgery, 50, 1119-1121. http://dx.doi.org/10.1016/0278-2391(92)90505-T

[17] Cmejrek, R., Coticchia, J. and Arnold, J. (2002) Presentation, Diagnosis, and Management of Deep-Neck Abscesses in Infants. Archives of Otolaryngology—Head \& Neck Surgery, 128, 1361-1364. http://dx.doi.org/10.1001/archotol.128.12.1361

[18] Chang, K.P., Chen, Y.L., Hao, S.P. and Chen, S.M. (2005) Ultrasound-Guided Closed Drainage for Abscesses of the Head and Neck. Otolaryngology_Head and Neck Surgery, 132, 119-124. http://dx.doi.org/10.1016/j.otohns.2004.08.004

[19] Peckitt, N.S., Fields, M.J. and Gregory, M.C. (1990) A Closed Drainage System for Head and Neck Sepsis. Journal of Oral and Maxillofacial Surgery, 48, 758-759. http://dx.doi.org/10.1016/0278-2391(90)90068-D

[20] Asai, N., Ohkuni, Y., Yamazaki, I., et al. (2013) Therapeutic Impact of CT-Guided Percutaneous Catheter Drainage in Treatment of Deep Tissue Abscesses. The Brazilian Journal of Infectious Diseases, 17, 483-486. http://dx.doi.org/10.1016/j.bjid.2012.12.008

[21] Duszak Jr., R.L., Levy, J.M., Akins, E.W., Bakal, C.W., et al. (2000) Percutaneous Catheter Drainage of Infected Intra- 
Abdominal Fluid Collections. American College of Radiology. ACR Appropriateness Criteria. Radiology, 215, 10671075.

[22] Van Sonnenberg, E., Wittich, G.R., Edwards, D.K., Casola, G., von Waldenburg, H.S., et al. (1987) Percutaneous Diagnostic and Therapeutic Interventional Radiologic Procedures in Children: Experience in 100 Patients. Radiology, 162, 601-605. http://dx.doi.org/10.1148/radiology.162.3.2949336 